Revue des sciences de l'eau

Journal of Water Science

\title{
Régionalisation des modules annuels et des régimes d'étiage du bassin hydrographique de la Moselle française : lien entre modèles régionaux Annual mean discharge and low flow regime regionalization of
the French Moselle catchment: link between regional models
}

\author{
G. Galéa et S. Canali
}

Volume 18, numéro 3, 2005

URI : https://id.erudit.org/iderudit/705562ar

DOI : https://doi.org/10.7202/705562ar

Aller au sommaire du numéro

Éditeur(s)

Université du Québec - INRS-Eau, Terre et Environnement (INRS-ETE)

ISSN

0992-7158 (imprimé)

1718-8598 (numérique)

Découvrir la revue

Citer cet article

Galéa, G. \& Canali, S. (2005). Régionalisation des modules annuels et des régimes d'étiage du bassin hydrographique de la Moselle française : lien entre modèles régionaux. Revue des sciences de l'eau / Journal of Water Science, 18(3), 331-352. https://doi.org/10.7202/705562ar

\section{Résumé de l'article}

Les modélisations régionales proposées sur les modules annuels et les débits moyens d'étiage des sous-bassins de la Moselle française devraient contribuer à l'amélioration des connaissances sur le fonctionnement physique actuel des hydrosystèmes. Elles s'inscrivent dans le contexte des directives de la loi Pêche et de la loi sur l'Eau et plus récemment de la Directive Cadre Européenne. Quarante neuf sous-bassins répartis en trois jeux ont permis de caler et valider un modèle régional des modules annuels et un modèle régional d'étiage. Dans le cas du bassin hydrographique de la Moselle française une certaine dépendance existe entre modèles régionaux dont la loi statistique choisie est la loi de Weibull à 2 paramètres. Une pseudo-dépendance est observée entre la loi régionale des modules annuels et la loi régionale des débits moyens d'étiage pour les années moyennes à sèches. Cette propriété va permettre en particulier l'usage d'une procédure simplifiée commune, établie à partir de la

connaissance de jaugeages épisodiques d'étiage, pour l'estimation des descripteurs de débit d'un sous-bassin non observé : le module médian /qa et le débit quotidien minimal médian $/ \mathrm{vcn}$. . Pour le modèle régional d'étiage un deuxième descripteur local est nécessaire. Il s'agit d'un temps caractéristique d'étiage du sous-bassin $\Delta$ e (j) permettant de généraliser le modèle à toute durée $d$. Le concept débit-durée-fréquence $Q d F$ appliqué aux étiages exploite la convergence observée des distributions de différentes durées $d$ et est indépendant de la loi fréquentielle choisie. Le caractère opérationnel de ces modélisations régionales dépend essentiellement de la précision d'estimation des descripteurs de débit du sous-bassin étudié /qa, pour les modules annuels et / $\mathrm{vcn}_{\mathrm{d}=1}$ pour les étiages. Ces descripteurs de débit ont été estimés selon deux approches : l'approche classique par régression multiple et selon une approche simple de recherche d'un coefficient de tendance $k$ entre jaugeages épisodiques d'étiage concomitants au sous-bassin étudié (pas ou peu d'observations) et au sous-bassin de référence (chronique de débit continue). Pour cela, un choix de cinq jaugeages d'étiage par an sur les douze dernières années en moyenne a été fait. Le descripteur de débit du sous-bassin étudié est ensuite déduit du produit de $k$ par le descripteur de débit du sous-bassin de

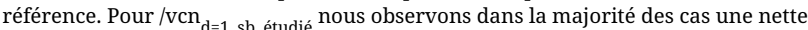
amélioration de l'estimation obtenue par régression, notamment une forte réduction des écarts les plus importants. Une similitude des classes de superficie entre sous-bassin étudié et sous-bassin de référence n'est pas exigée. La proximité géographique des sous-bassins semble donner de meilleurs résultats. En ce qui concerne le module médian /qa $\mathrm{sb}_{\text {étudié }}$, son estimation par régression multiple est assez performante. Parallèlement a cela, le coefficient $k$ de tendance permet, de même que pour, une estimation cohérente de /qa résultat un peu inattendu laisse supposer que la pseudo-dépendance observée entre modèles régionaux a bien une réalité physique. Nous avons insisté sur cette démarche "de régionalisation" nécessitant un faible investissement en mesures de débit des sous-bassins non observés par rapport au réseau national de suivi hydrométrique. Elle se présente à notre avis comme une alternative (ou complémentarité) intéressante aux méthodes de

régionalisation à bases géostatistiques : telles que l'identification du voisinage hydrologique homogène du sous-bassin étudié ou encore la prise en compte de l'effet structurant du réseau hydrographique dans la cartographie du descripteur de débit. L'ensemble des connaissances relative à cette recherche est repris dans un Système d'Information Géographique pour répondre éventuellement à la demande.
Ce document est protégé par la loi sur le droit d'auteur. L’utilisation des services d’Érudit (y compris la reproduction) est assujettie à sa politique d'utilisation que vous pouvez consulter en ligne.

https://apropos.erudit.org/fr/usagers/politique-dutilisation/ 


\title{
Régionalisation des modules annuels et des régimes d'étiage du bassin hydrographique de la Moselle française : lien entre modèles régionaux
}

\author{
Annual mean discharge and low flow regime \\ regionalization of the French Moselle catchment: \\ link between regional models
}

\author{
G. GALÉA* ${ }^{\star}$ S. CANALI
}

Reçu le 21 janvier 2004, accepté le 6 décembre $2004^{\star \star}$.

Regional modelling of the annual mean discharge and average low-flow discharge of the French Moselle sub-basins should improve our knowledge of its hydrosystem functioning. These models are part of the directives of the Water Act and Fishery Act and more recently of the European Framework Directive. Forty-nine sub-basins divided into three groups were used to calibrate and validate a regional model of the annual mean discharge and a regional model of low-flow discharge.

A two-parameter Weibull distribution was used to represent the regional models of the Moselle catchment. Results show a relative dependence between the regional models. A pseudo-dependence was observed between the regional annual mean discharge model and the regional average low-flow discharge model for the average years to the dry ones. This property allowed us to use a common simplified procedure, established from the low-flow episodic discharge measurements, to estimate the flow descriptors of an ungauged sub-basin, namely the median annual mean discharge $\overline{q a}$ and the median minimal daily low-flow discharge $\overline{v c n}_{d=1}$. For the low-flow regional model a second local descriptor was necessary, the characteristic time of lowflow discharges of the sub-basin $\Delta e(j)$, which allowed us to generalize the model for every duration (d). The QdF flow-duration-frequency concept, applied to the low-flow discharge, is independent of the selected frequency law and uses the observed convergence of the distributions of various durations (d). The operational character of these regional models depends strongly on the accuracy of the estimation of the flow descriptors of the studied sub-basin $\overline{q a}$ for the annual mean discharges and $\overline{v c n}_{d=1}$ for the low-

Cemagref-Lyon, Unité de Recherche Hydrologie Hydraulique, 3 bis, quai Chauveau, 69336 Lyon Cedex 09, France.

* Correspondance: gilles.galea@cemagref.fr

** Les commentaires seront reçus jusqu'au 30 juin 2006. 
flow discharges). These flow descriptors were estimated according to two approaches: a traditional approach based on multiple regression and a simple approach based on a trend coefficient $(k)$ calculated between low-flow episodic discharge measurements that are concomitant between the studied sub-basin (no or few observations) and the reference sub-basin (time series of continuous discharges). A choice of five low flow discharge measurements per year over the last twelve years (on average) was made. Knowing the flow descriptor of the reference sub-basin, the flow descriptor of the studied subbasin was then deduced from the trend coefficient $k$. For $\overline{v c n}_{d=1, \text { sb.étudié }}$ we observe in the majority of cases a significant improvement of the estimation obtained by regression, especially a net reduction of the most important variations. A similarity of the surface classes between the studied sub-basin and the reference sub-basin is not required. Geographical proximity of the subbasins seems to provide better results. The estimation of the median annual mean discharges $\overline{q a}_{\text {sb.étudié, }}$, using multiple regression was rather impressive. In addition, the trend coefficient $k$ established for the low-flow discharges allowed a coherent estimation of $\overline{v c n}_{d=1, \text { sb.étudie }}$ and $\overline{q a}_{\text {sb.étudié }}$ This quite unexpected result suggests that the observed pseudo-dependence between regional models has a physical reality.

The "regionalization" technique detailed herein does not require a significant investment in flow measurements of the ungauged sub-basin compared to the national hydrometric network. It is an interesting (or complementary) alternative to the regionalization approach based on geostatistical methods that, for instance, identify the homogeneous hydrological vicinity of each subbasin or take into account the structuring effect of the hydrographic network in the cartography of the flow descriptor.

Key words: regionalization, annual mean discharges, low flow, statistical QdF modelling.

\section{RÉSUMÉ}

Les modélisations régionales proposées sur les modules annuels et les débits moyens d'étiage des sous-bassins de la Moselle française devraient contribuer à l'amélioration des connaissances sur le fonctionnement physique actuel des hydrosystèmes. Elles s'inscrivent dans le contexte des directives de la loi Pêche et de la loi sur l'Eau et plus récemment de la Directive Cadre Européenne. Quarante-neuf sous-bassins répartis en trois jeux ont permis de caler et valider un modèle régional des modules annuels et un modèle régional d'étiage. Dans le cas du bassin hydrographique de la Moselle française une certaine dépendance existe entre modèles régionaux dont la loi statistique choisie est la loi de Weibull à 2 paramètres. Une pseudo-dépendance est observée entre la loi régionale des modules annuels et la loi régionale des débits moyens d'étiage pour les années moyennes à sèches. Cette propriété va permettre en particulier l'usage d'une procédure simplifiée commune, établie à partir de la connaissance de jaugeages épisodiques d'étiage, pour l'estimation des descripteurs de débit d'un sous-bassin non observé : le module médian $\overline{q a}$ et le débit quotidien minimal médian $\overline{v c n}_{d=1}$. Pour le modèle régional d'étiage un deuxième descripteur local est nécessaire. Il s'agit d'un temps caractéristique d'étiage du sous-bassin $\Delta e$ (j) permettant de généraliser le modèle à toute durée $d$. Le concept débit-durée-fréquence $Q d F$ appliqué aux étiages exploite la convergence observée des distributions de différentes durées $\boldsymbol{d}$ et est indépendant de la loi fréquentielle choisie. Le caractère opérationnel de ces modélisations régionales dépend essentiellement de la précision d'estimation des descripteurs de débit du sous-bassin étudié, $\overline{q a}$ pour les modules annuels et $\bar{v}_{d=1}$ pour les étiages. Ces descripteurs de débit ont été estimés selon deux approches : l'approche classique par régression multiple et selon une approche simple de recherche d'un coeffi- 
cient de tendance $k$ entre jaugeages épisodiques d'étiage concomitants au sous-bassin étudié (pas ou peu d'observations) et au sous-bassin de référence (chronique de débit continue). Pour cela, un choix de cinq jaugeages d'étiage par an sur les douze dernières années en moyenne a été fait. Le descripteur de débit du sous-bassin étudié est ensuite déduit du produit de $k$ par le des-

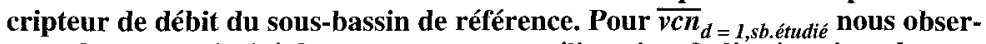
vons dans la majorité des cas une nette amélioration de l'estimation obtenue par régression, notamment une forte réduction des écarts les plus importants. Une similitude des classes de superficie entre sous-bassin étudié et sous-bassin de référence n'est pas exigée. La proximité géographique des sous-bassins semble donner de meilleurs résultats. En ce qui concerne le module médian $\overline{q a}_{s b . e ́ t u d i e ́}$, son estimation par régression multiple est assez performante. Parallèlement à cela, le coefficient $k$ de tendance permet, de

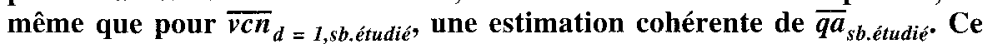
résultat un peu inattendu laisse supposer que la pseudo-dépendance observée entre modèles régionaux a bien une réalité physique. Nous avons insisté sur cette démarche « de régionalisation » nécessitant un faible investissement en mesures de débit des sous-bassins non observés par rapport au réseau national de suivi hydrométrique. Elle se présente à notre avis comme une alternative (ou complémentarité) intéressante aux méthodes de régionalisation à bases géostatistiques : telles que l'identification du voisinage hydrologique homogène du sous-bassin étudié ou encore la prise en compte de l'effet structurant du réseau hydrographique dans la cartographie du descripteur de débit. $L$ 'ensemble des connaissances relative à cette recherche est repris dans un Système d'Information Géographique pour répondre éventuellement à la demande.

Mots clés : régionalisation, module annuel, étiage, modélisation statistique QdF.

\section{1 - CONTEXTE HISTORIQUE}

La régionalisation des modules annuels (débits moyens annuels) et des régimes d'étiage des sous-bassins de la Moselle française (CANALI, 2002) s'inscrit dans le contexte de la Directive Cadre Européenne (DCE, 2000). Elle devrait contribuer à l'amélioration des connaissances sur le fonctionnement physique actuel des hydrosystèmes. Elle fait suite à différents travaux de recherche effectués dans le cadre des projets NOAH (2000) et PACTES (2001). Concernant plus précisément les étiages (GALÉA et al., 2000), nous avons bénéficié des travaux de JAVELLE (2001), JAVELLE et al. (2002) et de DusSAUX (2000). Plus récemment, les recherches menées par BLUM (2003) et le développement d'un Système d'Information Géographique par VANDERBECQ (2003) ont nécessité d'homogénéiser l'information collectée et élaborée lors des différentes recherches. Notre propos est donc une présentation actualisée des principaux résultats de modélisation obtenus par CANALI (2002) avec en particulier l'identification d'un lien entre loi régionale des modules annuels et loi régionale des étiages. 


\section{2 - PRÉSENTATION DU RÉSEAU D'OBSERVATION}

Nous nous sommes référencés aux trois jeux de sous-bassins constitués à partir des 59 sites inventoriés lors des études sur les crues, dans le cadre des projets NOAH et PACTES (figure 1). Le choix des deux premiers jeux de sousbassins a privilégié à la fois des écoulements peu influencés et indépendants. Le troisième jeu concerne des sous-bassins dont l'écoulement peut être indépendant ou non (sous-bassins emboîtés) ainsi que peu ou prou influencé (éclusées).

Au total 49 sous-bassins ont été retenus selon divers critères de sélection : faibles périodes de lacune, chronique de durée suffisante, etc. (tableau 1).

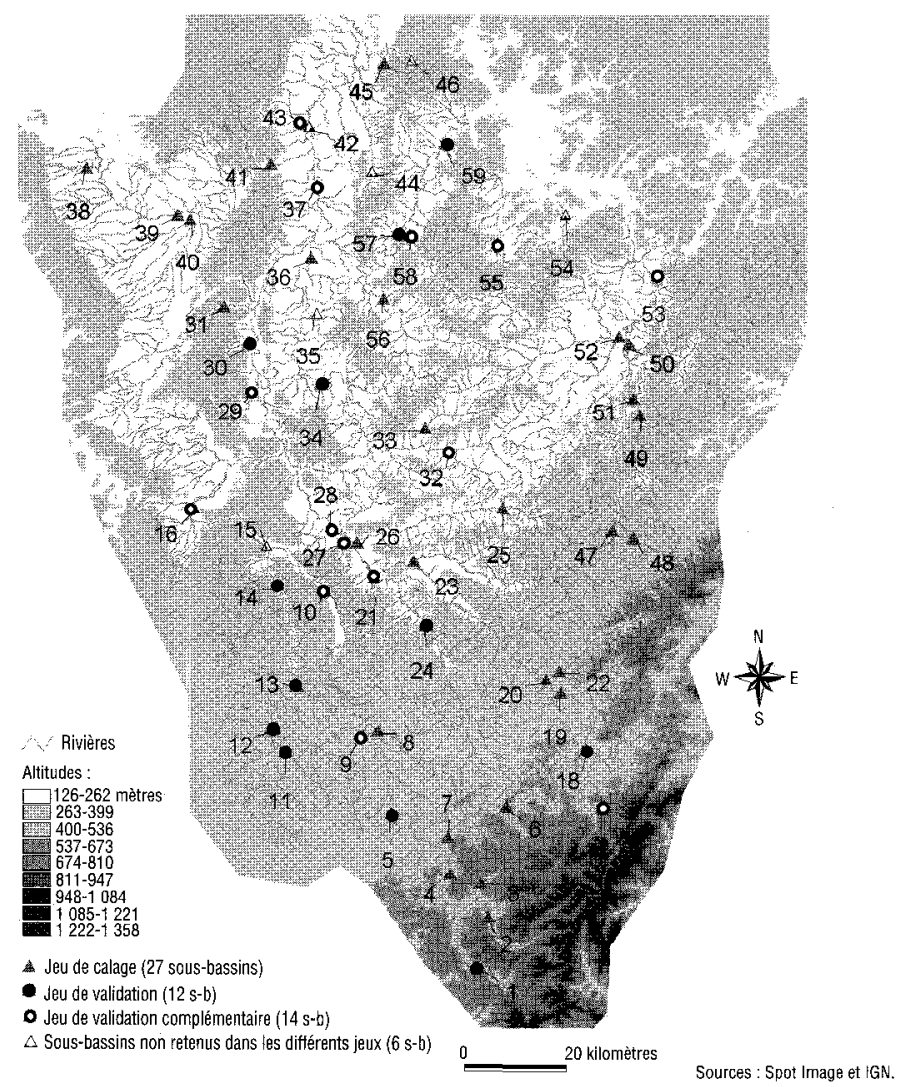

Figure 1 Situation des stations hydrométriques.

Location of the hydrometric stations. 
Tableau 1 Présentation des trois jeux de sous-bassins.

Table 1 Presentation of the three sub-basins sets.

\begin{tabular}{|c|c|c|c|c|c|c|c|}
\hline \multicolumn{4}{|c|}{$\begin{array}{l}\text { Jeu de calage } \\
23 \text { sous-bassins }\end{array}$} & \multicolumn{2}{|c|}{$\begin{array}{l}\text { Jeu de validation } \\
12 \text { sous-bassins }\end{array}$} & \multicolumn{2}{|c|}{$\begin{array}{c}\text { Jeu de validation complémentaire } \\
14 \text { sous-bassins } \\
\end{array}$} \\
\hline $\begin{array}{c}N^{\circ} \\
\text { Sous-bassin } \\
\end{array}$ & $\begin{array}{c}\text { Superficie } \\
\left(\mathrm{km}^{2}\right)\end{array}$ & $\begin{array}{c}N^{\circ} \\
\text { Sous-bassin } \\
\end{array}$ & $\begin{array}{c}\text { Superficie } \\
\left(\mathrm{km}^{2}\right)\end{array}$ & $\begin{array}{c}N^{\circ} \\
\text { Sous-bassin }\end{array}$ & $\begin{array}{l}\text { Superficie } \\
\left(\mathrm{km}^{2}\right)\end{array}$ & $\begin{array}{c}N^{\circ} \\
\text { Sous-bassin } \\
\end{array}$ & $\begin{array}{c}\text { Superficie } \\
\left(\mathrm{km}^{2}\right)\end{array}$ \\
\hline 2 & 186 & 39 & 406 & 1 & 153 & 9 & 103 \\
\hline 3 & 65,6 & 41 & 1252 & 5 & 1222 & 10 & 1985 \\
\hline 4 & 633 & 45 & 56,2 & 11 & 121 & 16 & 3367 \\
\hline 6 & 95,2 & 47 & 77 & 12 & 404 & 17 & 66,5 \\
\hline 7 & 357 & 48 & 90,4 & 13 & 64 & 21 & 2295 \\
\hline 8 & 146 & 49 & 142 & 14 & 967 & 27 & 21,6 \\
\hline 19 & 152 & 50 & 878 & 18 & 366 & 28 & 2787 \\
\hline 20 & 724 & 52 & 406 & 24 & 494 & 29 & 226 \\
\hline 22 & 118 & 56 & 16,2 & 30 & 38 & 32 & 363 \\
\hline 23 & 566 & & & 34 & 930 & 37 & 9395 \\
\hline 25 & 69,1 & & & 57 & 492 & 43 & 83.6 \\
\hline 31 & 344 & & & 59 & 1145 & 53 & 1757 \\
\hline 33 & 145 & & & & & 55 & 55,7 \\
\hline 36 & 1230 & & & & & 58 & 371 \\
\hline
\end{tabular}

\section{3 - RÉGIONALISATION DES MODULES ANNUELS}

\subsection{Loi locale et loi régionale}

Pour chacun des 49 sous-bassins retenus est ajustée sur l'échantillon des modules annuels observés qa une loi de Weibull à 2 paramètres (1).

$$
Q A_{L}\left(U_{F}\right)=\beta U_{F}^{1 / \alpha}
$$

avec :

$U_{F}=-\ln (1-F)$

$\beta$ : paramètre d'échelle, $\left(\mathrm{m}^{3} / \mathrm{s}\right)$

$\alpha$ : paramètre de forme

$F_{i}=\frac{i-0,3}{n+0,4}$, probabilité au non-dépassement, $i$ : rang et $n:$ nombre d'années d'observation.

Pour chaque sous-bassin du jeu de calage, la loi locale adimensionnelle est obtenue en normant le paramètre d'échelle $\beta$ par la médiane de l'échantillon constitué $\overline{q a}$, soit $\beta^{*}=\frac{\beta}{\overline{q a}}$.

Les paramètres régionaux sont calculés en prenant la moyenne pondérée par le nombre d'années d'observation $(n)$ des paramètres $\alpha$ et $\beta^{\star}$ ajustés sur les $s$ sites (2). 


$$
\alpha R=\frac{\sum_{m=1}^{s=23}\left(\alpha_{m} \cdot n_{m}\right)}{\sum_{m=1}^{s=23} n_{m}} \quad \beta R=\frac{\sum_{m=1}^{s=23}\left(\beta_{m}^{*} \cdot n_{m}\right)}{\sum_{m=1}^{s=23} n_{m}}
$$

Les quantiles en un site cible pourront être déduits de l'équation du modèle régional (3) :

$$
Q A_{R}\left(U_{F}\right)=\left(\beta_{R} U_{F}^{\left(1 / \alpha_{R}\right)}\right) \overline{q a}_{\text {site cible }}
$$

avec :

$$
\beta_{R}=1,08 \text { et } \alpha_{R}=3,79
$$

Pour vérifier la robustesse du modèle régional (3) nous avons effectué un test d'erreur quadratique moyen (4), tous quantiles confondus, entre ajustements local et régional, pour chacun des jeux de s sous-bassins. Pour ce test nous considérons $\overline{q a}_{\text {site cible }}$ connu, grâce aux observations de débit (figure 2).

$$
E r_{j e u} \%=100 * \sqrt{\frac{\sum_{m=1}^{s} \sum_{i=1}^{n}\left(\frac{Q A_{m_{L}}\left(U_{F i}\right)-Q A_{m_{R}}\left(U_{F i}\right)}{Q A_{m_{L}}\left(U_{F i}\right)}\right)^{2}}{\sum_{m=1}^{s} n_{m}}}
$$

Pour les différents sous-bassins des trois jeux retenus (tableau 1) nous donnons une représentation de l'adéquation du modèle régional (3) pour le quantile «sec » (pour $F<0,5, T=1 / F)$ de période moyenne de retour $\mathrm{T}=5$ ans (figure 3 ).

Les deux plus fortes erreurs relatives sur le jeu de calage sont voisines de $32 \%$ et $36 \%$. Ces valeurs sont à tempérer compte tenu des faibles débits et des estimations obtenues qui sont cohérentes en ordre de grandeur, respectivement $0,355 \mathrm{~m}^{3} / \mathrm{s}$ (au lieu de $0,269 \mathrm{~m}^{3} / \mathrm{s}$ ) et $2,4 \mathrm{~m}^{3} / \mathrm{s}$ (au lieu de $1,76 \mathrm{~m}^{3} / \mathrm{s}$ ).
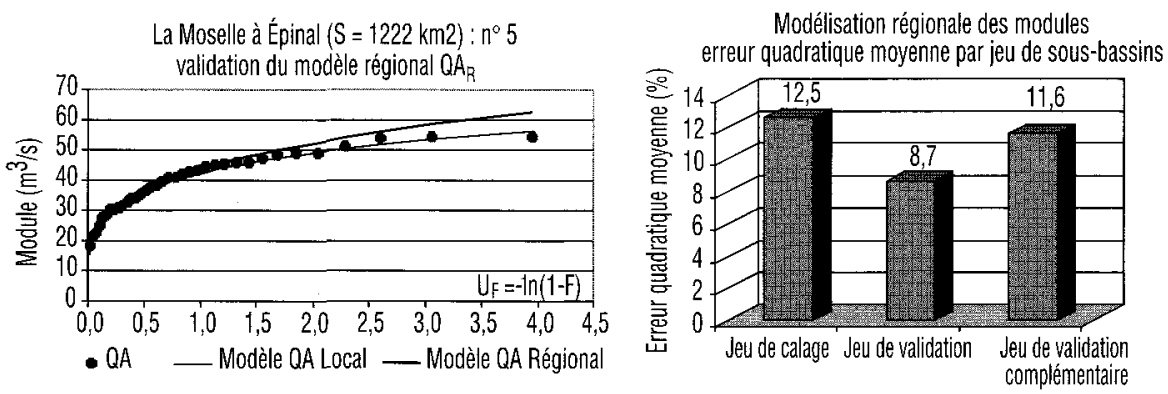

Figure 2 Modules annuels locaux et régionaux - Erreur quadratique moyenne. Local and regional annual mean discharges - average quadratic error. 

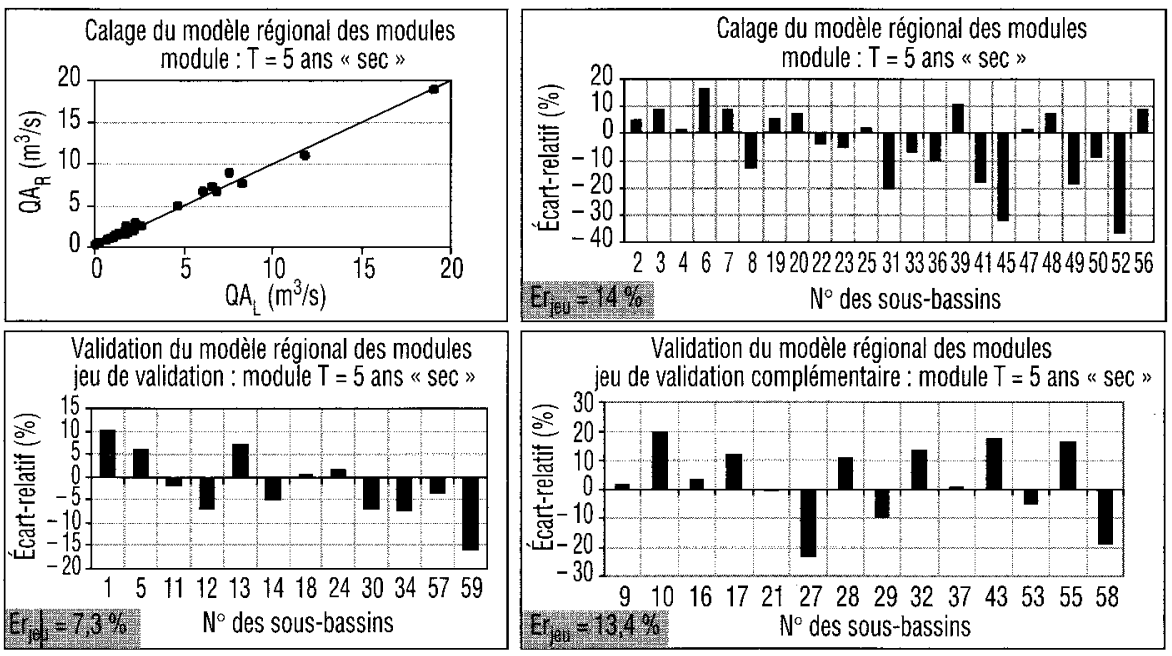

Figure 3 Validation du modèle régional pour le module « sec $» T=5$ ans.

Regional model validation for the "dry" annual mean discharge $(T=5$ years).

\subsection{Estimation des modules d'un sous-bassin non observé}

Pour estimer les quantiles du module annuel le modèle régional (3) est proposé avec (5), estimation du module médian $\overline{q a}$ en l'absence d'observations de débit :

$$
\overline{q a}_{\text {site cible }}=8,29 \cdot 10^{-6} \mathrm{~S}^{0,98} \mathrm{Zm}^{1,28}
$$

où :

\section{$Z m$ : altitude moyenne, $(m)$}

\section{$S$ : Superficie, $\left(\mathrm{km}^{2}\right)$}

La figure 4 permet de montrer l'adéquation du modèle d'estimation (5).

Pour ce qui concerne le jeu de validation complémentaire, sans les sous bassins 27, 29 et 43, l'erreur quadratique moyenne passe de $35 \%$ à $17 \%$. Pour le sous-bassin 43 la chronique de débit $Q(t)$ montre une influence majeure des éclusées sur l'écoulement annuel (GALÉA et al., 2001). Pour 27 et 29, compte tenu des faibles valeurs de débit l'estimation n'est pas d'un ordre de grandeur aberrant (tableau 2).

Tableau 2 qa médians observé et estimé associés à chacun des 3 sous-bassins

Table 2 Observed and estimated qa medians associated with each of the 3 sub-basins.

\begin{tabular}{|c|c|c|c|}
\hline \multicolumn{2}{|c|}{ Sous-bassin } & $\begin{array}{l}\text { qa médian observé } \\
\left(\mathrm{m}^{3} / \mathrm{s}\right)\end{array}$ & $\begin{array}{l}\text { qa médian estimé } \\
\qquad\left(\mathrm{m}^{3} / \mathrm{s}\right)\end{array}$ \\
\hline 27 & 21,6 & 0,12 & 0,22 \\
\hline 29 & 226 & 1,35 & 2,2 \\
\hline 43 & 83.6 & 2,25 & 0,98 \\
\hline
\end{tabular}


L'erreur quadratique moyenne obtenue pour le jeu de validation complémentaire comparativement à celles des jeux de calage et de validation est donc à relativiser. Nous verrons ultérieurement comment améliorer ces estimations du module médian qa à partir d'une démarche dérivée de la méthode des jaugeages épisodiques d'étiage (OBERLIN et al., 1973).
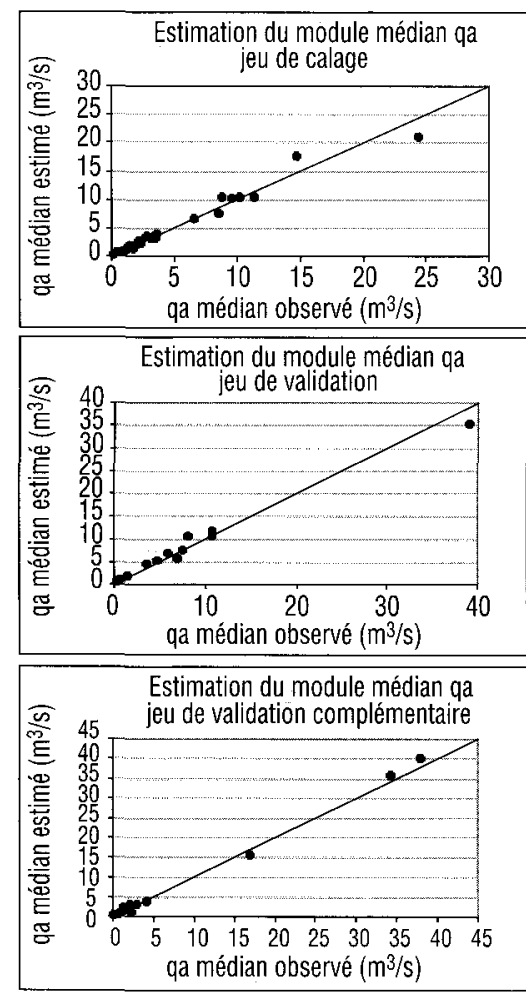
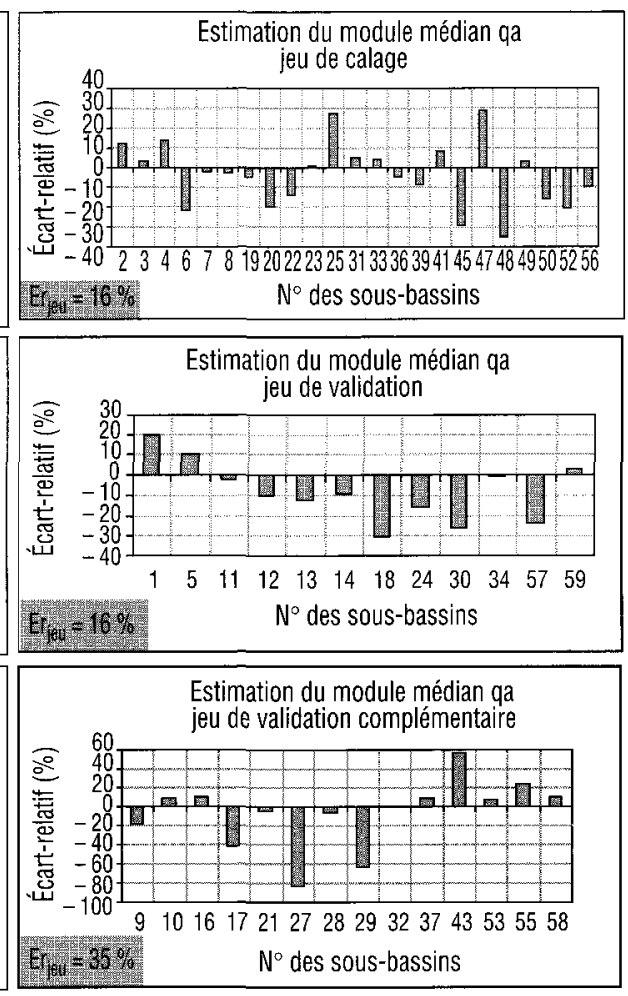

Figure 4 Validation du modèle d'estimation (5) de qa médian.

Validation of the qa median estimation model (5).

\section{4 - MODÉLISATION RÉGIONALE DES RÉGIMES D'ÉTIAGE}

\subsection{Variables hydrologiques}

En règle générale les étiages sont décrits à partir de deux variables de débit caractéristiques définies sur la saison-année et pour une durée continue $d$. II s'agit du débit moyen minimum $V C N d$ et du débit seuil minimum non dépassé QCNd (GALÉA et al., 1999). Cette dernière caractéristique est très utile pour certaines prises d'eau, pompages en rivières, de tirant d'eau à respecter pour la protection des écosystèmes aquatiques ; elle est cependant fortement influencée par les pluies d'été. 


\subsection{Modélisation débit-durée-fréquence (QdF) locale}

La modélisation $\mathrm{QdF}$ du régime d'étiage en chaque site observé utilise la propriété de convergence des distributions relatives aux différentes durées $d$ étudiées (figure 5). Pour les étiages, comme pour les modules annuels, c'est la loi de Weibull à deux paramètres (6) qui a été choisie (CANALI, 2002).

$$
V C N_{d}\left(U_{F}\right)=\beta_{d} U_{F}^{1 / \alpha_{d}}
$$

Nous faisons ci-après quelques rappels sur la démarche de modélisation (GALÉA et al., 2000). Elle consiste tout d'abord à consolider sur les quantiles expérimentaux des échantillons constitués une loi de distribution indépendante de la durée $d$. Cette dernière est ensuite associée au quantile expérimental médian quotidien pour obtenir la loi théorique consolidée des débits quotidiens. Le passage de la distribution de $d=1 j$ aux autres distributions de durée $d$ est possible grâce à une fonction de généralisation de la durée $d$ indépendante de $U_{F}$, calée sur ces mêmes échantillons.

L'ajustement consolidé est établi à partir de l'ensemble des échantillons de durée $d_{1}$ retenus (figure 5). Dans un premier temps et pour chaque durée $d_{l}$ les quantiles expérimentaux de rang $i$ sont normés par la médiane de l'échantillon correspondant : $v c n_{d_{l}}^{*}(i)=\frac{v c n_{d_{l}}(i)}{v c n_{d_{l}}}$,

Puis sur l'échantillon $v c n^{*}(i)=\frac{1}{4} \sum_{i=1}^{4} v c n_{d_{l}^{*}}^{*}(i)$ est ajustée la loi locale consolidée d'équation (7) :

$$
\operatorname{VCN}^{*}\left(U_{F}\right)=\beta^{c} U_{F}^{1 / \alpha C}
$$

Les quantiles théoriques de durée $d_{p}$, exprimés en $\mathrm{m}^{3} / \mathrm{s}$, seront déduits de l'expression (8) :

$$
V C N_{d_{l}}\left(U_{F}\right)=\beta^{c} U_{F}^{1 / \alpha^{c}} \overline{v C n_{d_{l}}}
$$

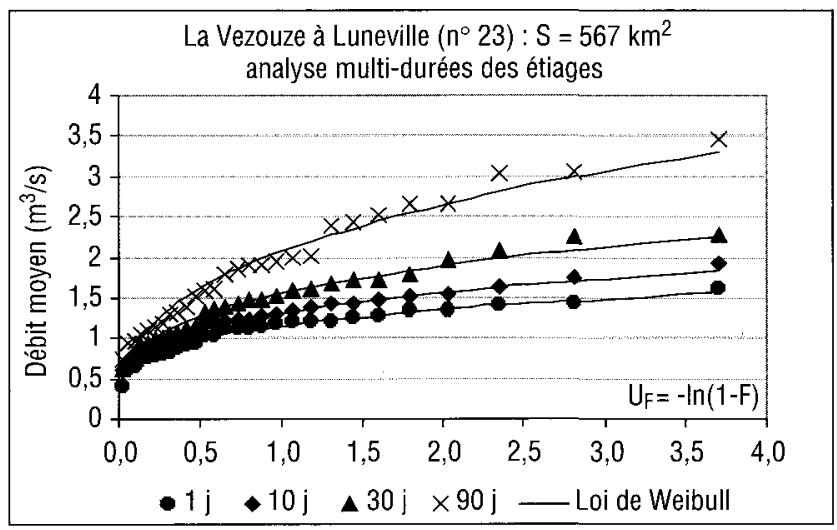

Figure 5 Loi de Weibull ajustée sur les échantillons vcn $\mathrm{d}_{\mathrm{d}}$.

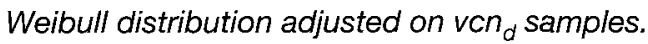


L'expression (8) peut être généralisée avec la durée $d$. Pour cela une fonction linéaire $Z(d)=a(d-1)+1$, indépendante de $U_{F}$, est calée sur l'ensemble de l'information échantillonnée réduite: $\frac{v c n_{d_{l}}(i)}{v c n_{d=1}(i)}$, où $i$ privilégie des plages de fréquences expérimentales proches de $F=0,5$, comprises entre 3 ans « sec » et 3 ans " humide " (pour $F>0,5(T=1 /(1-F)$ ). Le modèle $Q d F$ local $(L)$ d'étiage, généralisé avec $d$ et $U_{F}$, aura pour équation (9) :

où :

$$
V C N_{L}\left(d, U_{F}\right)=\beta^{c} U_{F}^{1 / \alpha c} \overline{v c n}_{\mathrm{d}=1}\left(\frac{d-1}{\Delta e}+1\right)
$$

$\Delta e=1 / \alpha:$ descripteur assimilé à un temps, durée caractéristique d'étiage du sous-bassin

Pour tester la pertinence des modélisations locales effectuées nous donnons pour chaque jeu de sous-bassin l'erreur quadratique moyenne (10) entre ajustement direct (6) et modèle QdF local (9). Pour ce calcul 5 durées sont considérées, les 4 de la figure 5 qui ont servi au calage du modèle QdF local et une extrapolation du modèle à la durée de 180 jours (tableau 3 ).

$$
E r_{j e u} \%=100 \sqrt{\frac{\sum_{m=1}^{s} \sum_{i=1}^{n} \sum_{l=1}^{5}\left(\frac{V C N_{m_{d_{i}}}\left(\mathrm{U}_{\mathrm{F} i}\right)-V C N_{m L}\left(d_{l}, U_{F i}\right)}{V C N_{m_{d_{l}}}\left(\mathrm{U}_{\mathrm{F} i}\right)}\right)^{2}}{5 \sum_{m=1}^{s} n_{m}}}
$$

Tableau 3 Modélisation locale - Erreur quadratique moyenne.

Table 3 Local modelling - average quadratic error.

\begin{tabular}{ccc}
\hline & Erreur quadratique moyenne (10) \\
\hline Jeu de calage & Jeu de validation & Jeu de validation complémentaire \\
\hline $11,7 \%$ & $10,3 \%$ & $10,5 \%$ \\
\hline
\end{tabular}

\subsection{Modélisation QdF régionale}

Les paramètres régionaux sont estimés à partir d'une moyenne pondérée, par le nombre d'années d'observation, des paramètres consolidés de la loi de weibull (7) des s sites du jeu de calage.

Un modèle régional $(R)$ est proposé pour estimer les quantiles d'étiage en un site cible (11):

$$
\left.V C N_{R}\left(d, U_{F}\right)=\beta_{R} U_{F}^{(1 / \alpha}\right) \overline{v C n}_{d-1, \text { site cible }}\left(\frac{d-1}{\Delta e_{\text {site cible }}}+1\right)
$$

avec :

$\alpha_{R}=2,697$

$\beta_{R}=1,184$ 
où :

$\overline{v c n}_{d=1 \text {, site cible }}=$ débit quotidien minimal médian, déduit de la loi ${ }^{1}$ ajustée(6), ou estimé (12)

$\Delta e_{\text {site }}=$ durée caractéristique d'étiage du sous-bassin, calée sur l'ensemble des échantillons de durée d constitué sur la saison d'étiage, ou estimé (13)

$$
\begin{gathered}
\overline{V C n}_{d=1, \text { site cible }}=2,05 \cdot 10^{-6} \mathrm{~S}^{0,92} \mathrm{Zm}^{1,23} \\
\Delta e_{\text {site cible }}=441 \mathrm{~S}^{-0,163} \mathrm{Ag}^{0,6}\left(\mathrm{Ag}: \mathrm{S}_{\text {Agriculture }} / \mathrm{S}_{\text {Bassin }}\right)
\end{gathered}
$$

De même que pour la modélisation QdF locale, la modélisation QdF régionale peut être éprouvée. Dans un premier temps, la comparaison des quantiles de toutes fréquences confondues et pour chaque jeu de sous-bassins porte entre la loi locale consolidée des débits quotidiens (8) et la loi régionale des débits quotidiens (11). Les erreurs quadratiques moyennes obtenues sont présentées au tableau 4.

Tableau 4 Erreur quadratique moyenne - entre (8) et (11) avec $\mathrm{d}=1$.

Table $4 \quad$ Average quadratic error - between (8) and (11) with $d=1$.

\begin{tabular}{ccc}
\hline \multicolumn{2}{c}{ Erreur quadratique moyenne - entre quantiles quotidiens modélisés selon (8) et selon (11) } \\
\hline Jeu de calage & Jeu de validation & Jeu de validation complémentaire \\
\hline $13,2 \%$ & $7,4 \%$ & $14,5 \%$ \\
\hline
\end{tabular}

Dans un deuxième temps, nous nous sommes intéressés aux quantiles quinquennaux « sec " et quinquennaux « humide " relatifs à 5 durées. Cette période de retour 5 ans est un des référentiels des services gestionnaires de la ressource en eau. Au tableau 5 est présentée l'erreur quadratique moyenne par jeu de sous-bassins et pour tous quantiles quinquennaux de durées $d$ confondus : $1 j, 10 j, 30 j, 90 j$ et $180 j$. L'estimation régionale (11) est comparée à (6) ou à (9).

Tableau 5 Erreur quadratique moyenne selon (11) et par rapport à (6) ou (9).

\begin{tabular}{|c|c|c|c|c|}
\hline $\begin{array}{c}\text { Jeu } \\
\text { de sous-bassins }\end{array}$ & \multicolumn{2}{|c|}{$\begin{array}{l}\text { Quantiles } 5 \text { ans « sec » } \\
\text { Erreur quadratique moyenne }(\%) \\
(11) /(6) \quad(11) /(9)\end{array}$} & \multicolumn{2}{|c|}{$\begin{array}{l}\text { Quantiles } 5 \text { ans « humide " } \\
\text { Erreur quadratique moyenne }(\%) \\
(11) /(6) \quad(11) /(9)\end{array}$} \\
\hline Calage & 17 & 12,4 & 15,7 & 14,1 \\
\hline Validation & 12,2 & 7,1 & 11,6 & 6,7 \\
\hline Validation complémentaire & 14,5 & 10,9 & 15,5 & 12,8 \\
\hline
\end{tabular}
Table 5 Average quadratic error according to (11) and compared to (6) or (9).

Les différents résultats présentés montrent que le modèle QdF régional (11) donne pour un site cible donné, dont les descripteurs de régime $\overline{V C n}_{d=1, \text { site cible }}$ et $\Delta$ e sont connus avec précision (déduits des observations), une bonne estimation des quantiles d'étiage. L'erreur d'estimation est d'un ordre de grandeur

1. Pour un nombre d'années significatif, le quantile médian expérimental est proche du théorique. 
comparable entre quantiles quinquennaux «sec » et quantiles quinquennaux " humide" (tableau 5). Elle est en moyenne systématiquement plus faible entre le modèle $\mathrm{QdF}$ régional et le modèle $\mathrm{QdF}$ local. Cela pourrait être lié au caractère d'homogénéisation commun aux modélisations $\mathrm{QdF}$, ce qui n'est pas le cas avec les ajustements relatifs à des valeurs discrètes de la durée $d(6)$.

\subsection{Quantiles de débit seuil}

La caractéristique d'étiage en $Q C N d$, souvent influencée par les pluies d'été, revêt un intérêt moindre que celle en $V C N d$ plus pertinente pour renseigner sur la ressource disponible en période critique. La démarche de modélisation QdF locale et régionale aurait pu être réitérée de manière analogue à partir des ajustements directs (14) sur les échantillons constitués.

$$
Q C N_{d}\left(U_{F}\right)=\beta_{d} U_{F}^{1 / \alpha_{d}}
$$

Ce travail n'est pas nécessaire grâce à la propriété (15) mise en évidence par GALÉA et al. (2000).

$$
\operatorname{QCN}\left(d, U_{F}\right)=\frac{\partial\left((d-1) \vee C N\left(d, U_{F}\right)\right)}{\partial d}
$$

Soit, les modèles QdF local (16) et régional (17) des débits seuils d'étiage :

$$
\begin{gathered}
Q C N_{L}\left(d, U_{F}\right)=\beta^{c} U_{F}^{1 / \alpha} \overline{v c n}_{\mathrm{d}=1}\left(2 \frac{d-1}{\Delta e}+1\right) \\
Q C N_{R}\left(d, U_{F}\right)=\beta_{R} U_{F}^{1 / \alpha} \overline{V C n}_{\mathrm{d}=1 \text {, site cible }}\left(2 \frac{d-1}{\Delta e_{\text {site cible }}}+1\right)
\end{gathered}
$$

Les durées préconisées ne doivent pas dépasser une dizaine de jours à un mois, au-delà l'influence de la pluie n'est pas négligeable. Au tableau 6 sont données les erreurs quadratiques moyennes entre modélisation QdF (16) ou (17) et ajustement direct (14) et pour les durées $d$ de 10 j et $30 \mathrm{j}$. Seuls 6 sous-bassins ont été étudiés, pris parmi les sous-bassins des 3 jeux retenus (CANALI, 2002).

Tableau 6 Quantiles de débits seuils - Erreur quadratique moyenne.

Table 6 Threshold discharge quantiles - average quadratic error.

\begin{tabular}{cccccc}
\hline \multirow{2}{*}{$\begin{array}{c}\text { Jeu } \\
\text { de sous-bassins }\end{array}$} & $\begin{array}{c}\mathbf{N}^{\circ} \\
\text { sous-bassin }\end{array}$ & \multicolumn{4}{c}{$\begin{array}{c}\text { Erreur quadratique moyenne } \\
\text { tous quantiles confondus (\%) }\end{array}$} \\
\cline { 3 - 6 } & & \multicolumn{2}{c}{$\mathbf{( 1 6 ) / ( 1 4 )}$} & \multicolumn{2}{c}{$\mathbf{( 1 7 ) / ( 1 4 )}$} \\
\cline { 3 - 6 } & 33 & 2,9 & 10,5 & 9,2 & $\mathbf{3 0 ~ j}$ \\
\hline Calage & 3 & 5,6 & 26,5 & 8,3 & 23,2 \\
& 11 & 8,7 & 7,4 & 20,6 & 16,3 \\
Validation & 5 & 4,4 & 15,1 & 7,3 & 18,1 \\
& 29 & 14,3 & 22,4 & 24,7 & 22,7 \\
Validation & 10 & 1,5 & 12,2 & 9,6 & 21,5 \\
complémentaire & 10 & & & & $\mathbf{3 0} \mathbf{j}$ \\
\hline
\end{tabular}

L'incertitude liée au modèle régional (17) reste plus élevée mais d'un ordre de grandeur satisfaisant par rapport à celle du modèle local (16). 


\section{5 - LIEN ENTRE MODÈLES RÉGIONAUX DES MODULES ANNUELS ET DES RÉGIMES D'ÉTIAGE}

Ce lien a été identifié en cherchant à améliorer l'estimation du descripteur de débit d'étiage local (12), à partir d'une approche simplifiée de la méthode des jaugeages épisodiques (OBERLIN et al., 1973). II est intéressant de la mentionner bien qu'en marge des méthodes de régionalisation, à bases géostatistiques notamment (Developments in water science 48, 2003). Elle représente pour nous une alternative (ou une complémentarité) des approches de voisinage hydrologique homogène (BLUM, 2003) ou encore de prise en compte de l'effet structurant du réseau hydrographique dans la cartographie du descripteur de débit (SAUQUET et al., 2003). La transposition de ces approches des crues aux étiages est en cours et pour l'instant les résultats obtenus par BLUM (2003) sont peu probants.

\subsection{Amélioration de l'estimation du descripteur de débit $\overline{\mathrm{vcn}}_{d=1 \text {, site cible }}$}

La démarche nécessite de disposer de mesures épisodiques d'étiage du sous-bassin étudié ainsi que des débits concomitants du sous-bassin de référence dont les basses eaux sont connues avec précision et continûment. Le débit quotidien médian du bassin de référence est donc statistiquement connu. Nous avons considéré en moyenne les 12 dernières années et 5 mesures épisodiques d'étiage réparties sur la saison d'étiage (figure 6). Le principe de la démarche est présentée pour les sous-bassins $n^{\circ} 1$ et $n^{\circ} 2$ dont les caractéristiques sont précisées antérieurement (figure 1 et tableau 1).

Sous-bassin de référence : $\mathrm{n}^{\circ} 2$
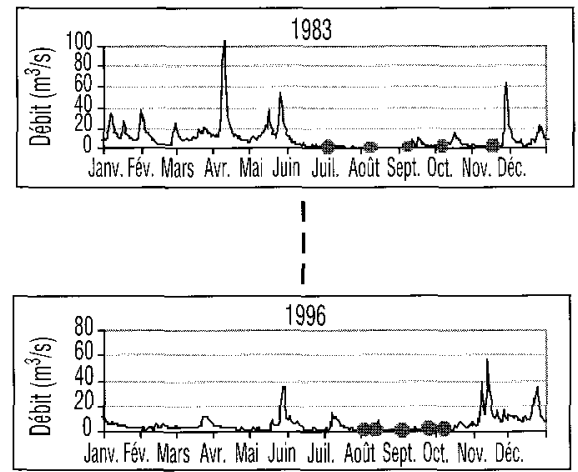

Sous-bassin étudié : $\mathrm{n}^{\circ} 1$

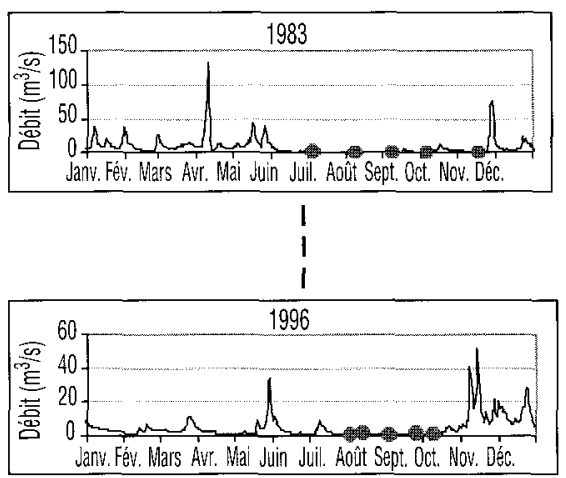

Figure 6 Choix des débits d'étiage concomitants.

Choice of concomitant low flow discharges. 
Les débits sont cumulés et représentés graphiquement (figure 7). Le coefficient $k$ de la droite de tendance permet ensuite d'estimer le descripteur du sous-bassin étudié $n^{\circ} 1$ connaissant celui du sous-bassin de référence $n^{\circ} 2$.

Cette estimation déduite des jaugeages épisodiques peut être ensuite comparée à la valeur observée. Au tableau 7 nous avons considéré les sous-bassins du jeu de validation et à chacun d'eux nous avons affecté un sous-bassin de référence pris parmi les sous-bassins du jeu de calage. Pour le sous-bassin étudié, le sous-bassin de référence peut être proche ou géographiquement éloigné (se référer à la figure 1).

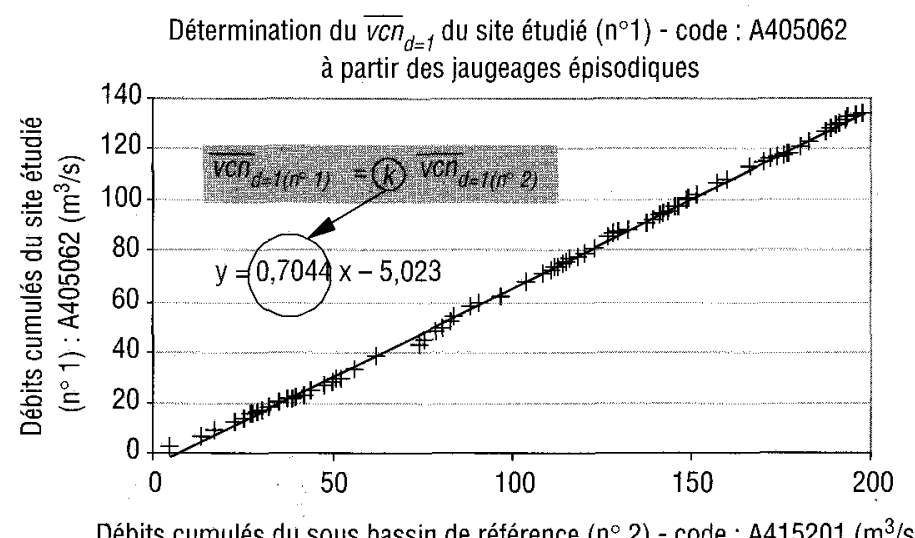

Figure 7 Définition du coefficient $K$ et du descripteur de régime du sous-bas$\sin n^{\circ} 1$.

Definition of coefficient $K$ and of regime descriptor of sub-basin $n^{\circ} 1$. 
Tableau 7 Estimation du descripteur de régime $\overline{v c n}_{d=1}$ selon (12) ou selon jaugeages épisodiques. A4 $15201^{*}$ : sous-bassin de référence de proximité.

A614201 : étiage peu influencé.

Table 7 Estimation of regime descriptor $\overline{V C n}_{d=1}$ according to (12) or to the episodic gauging. A415201*: proximity of the reference sub-basin.

A614201: a small influence on the low-flow.

\begin{tabular}{|c|c|c|c|c|c|c|c|c|c|c|c|c|}
\hline \multicolumn{4}{|c|}{$\begin{array}{c}\text { Sous-bassin de référence } \\
\text { (jeu de calage) }\end{array}$} & \multirow[b]{2}{*}{ Code } & \multirow[b]{2}{*}{$\mathbf{N}^{\circ}$} & \multirow[b]{2}{*}{$\underset{\left(\mathrm{km}^{2}\right)}{\mathrm{S}}$} & \multicolumn{4}{|c|}{$\begin{array}{l}\text { Sous-bassin étudié } \\
\text { (jeu de validation) }\end{array}$} & \multirow[b]{2}{*}{$\begin{array}{c}\text { Écart relatif } \\
([1]-[2]) /[1] \\
(\%)\end{array}$} & \multirow[b]{2}{*}{$\begin{array}{c}\text { Écart relatif } \\
([1]-[3]) /[1] \\
(\%)\end{array}$} \\
\hline Code & $\mathbf{N}^{\circ}$ & $\underset{\left(\mathbf{k m}^{2}\right)}{\mathrm{S}}$ & $\begin{array}{c}\overline{v c n}_{d=1} \\
\text { observé } \\
\left(\mathrm{m}^{3} / \mathrm{s}\right)\end{array}$ & & & & $\begin{array}{c}\overline{v c n}_{d=1}[1] \\
\text { observé } \\
\left(\mathrm{m}^{3} / \mathrm{s}\right)\end{array}$ & $\begin{array}{c}\overline{v G n}_{d=1}[2] \\
\text { estimé } \\
\text { par régression (12) } \\
\left(\mathrm{m}^{3} / \mathrm{s}\right)\end{array}$ & $\begin{array}{c}\overline{V C n}_{d=1}[3] \\
\text { estimé } \\
\text { par jaugeages } \\
\left(\mathrm{m}^{3} / \mathrm{s}\right)\end{array}$ & K & & \\
\hline A415201* & 2 & 186 & 0,75 & A405062 & 1 & 153 & 0,59 & 0,72 & 0,53 & 0,70 & -22 & 10 \\
\hline A436202* & 7 & 357 & 1,45 & A425064 & 5 & 1222 & 5,15 & 3,98 & 5,60 & 3,86 & 23 & -9 \\
\hline A444201 & 8 & 145 & 0,243 & A524201 & 11 & 116 & 0,196 & 0,214 & 0,200 & 0,82 & -9 & -2 \\
\hline A444201 & 8 & 145 & 0,243 & A525101 & 12 & 399 & 0,782 & 0,657 & 0,748 & 3,08 & 16 & 4 \\
\hline A444201 & 8 & 145 & 0,243 & A530301 & 13 & 64 & 0,107 & 0,117 & 0,117 & 0,48 & -9 & -9 \\
\hline A614201 & 19 & 152 & 0,957 & A605102 & 18 & 371 & 1,59 & 1,31 & 1,90 & 1,99 & 18 & -20 \\
\hline A656111 & 23 & 567 & 1,06 & A543101 & 14 & 963 & 1,11 & 1,40 & 1,26 & 1,19 & -26 & 14 \\
\hline A656111* & 23 & 567 & 1,06 & A673122 & 24 & 499 & 1,36 & 0,847 & 1,36 & 1,28 & 38 & 0 \\
\hline A735201* & 31 & 366 & 0,449 & A721301 & 30 & 38,4 & 0,048 & 0,069 & 0,034 & 0,08 & -44 & 29 \\
\hline A764201 & 33 & 144 & 0,302 & A782101 & 34 & 928 & 1,03 & 0,919 & 1,31 & 4,33 & 11 & -27 \\
\hline A788101 & 36 & 1273 & 1,23 & A975201 & 57 & 495 & 0,434 & 0,564 & 0,503 & 0,41 & -30 & -16 \\
\hline A885301 & 45 & 47 & 0,058 & A994201 & 59 & 1148 & 1,72 & 1,25 & 1,36 & 23,5 & 27 & 21 \\
\hline
\end{tabular}


Au vu de ces résultats nous pouvons retenir 5 points :

- dans la majorité des cas nous observons une amélioration de l'estimation donnée par (12), dans le cas contraire nous ne remarquons pas de dégradation significative de l'estimation ;

- les écarts relatifs les plus importants sont nettement réduits ;

- l'amélioration constatée ne dépend pas de la similitude des classes de superficie entre sous-bassins étudiés et sous-bassins de référence. Nous citerons en particulier (tableau 7) les sous-bassins étudiés $n^{\circ} 11, n^{\circ} 12$ et $n^{\circ} 13$ dont le sous-bassin de référence est le $n^{\circ} 8$;

- la proximité (toute relative) du sous-bassin de référence peut être un facteur d'amélioration de l'estimation. Pour trois des sous-bassins déjà étudiés (tableau 7) on constate une amélioration de l'estimation (tableau 8) à partir de sous-bassins de référence de proximité pris parmi ceux du jeu de validation complémentaire. Le test n'est cependant pas significatif compte tenu du petit nombre de sous-bassins considérés. Ceci dit, la situation géographique (proche ou éloignée) du sous-bassin de référence n'induit pas a priori d'incohérence quant à l'ordre de grandeur d'estimation du descripteur de débit (tableaux 7 et 8);

- nous noterons que parmi les sous-bassins de référence et les sous-bassins étudiés certains ont leur écoulement influencé en étiage. Lorsqu'il s'agit du sous-bassin de référence de proximité $n^{\circ} 17$ (tableau 8) au lieu de $n^{\circ} 19$ (tableau 7), tous deux influencés, nous noterons une nette amélioration de l'estimation du descripteur du sous-bassin étudié $\mathrm{n}^{\circ} 18$ dont l'erreur relative passe de $-20 \%$ à $4 \%$. Lorsqu'il s'agit de sous-bassins étudiés influencés (tableau 7) la démarche impliquant un sous-bassin de référence non influencé permet d'améliorer ou de confirmer l'estimation par régression (12). La considération d'un écoulement influencé du sousbassin de référence ou du sous-bassin étudié ne semble donc pas incompatible pour la tendance $(k)$ recherchée.

Ces résultats encourageants constituent quelques éléments de réflexion (notamment en matière d'influence) sur notre démarche qui mérite d'être poursuivie, parallèlement à des approches géostatistiques dont notre expérience récente n'a pas été pour l'instant concluante (BLUM, 2003). 
Tableau 8 Estimation du descripteur de régime $\overline{v C n}_{d=1}$ selon (12) ou selon jaugeages épisodiques. A511061* : sous-bassin de référence de proximité.

A600101 : étiage peu influencé.

Table 8 Estimation of regime descriptor $\overline{v C n}_{d=1}$ according to (12) or to the episodic gauging. $A 511061^{*}$ : proximity of the reference sub-basin. A600101: a small influence on the low-flow.

Sous-bassin de référence

(jeu de validation complémentaire)

\begin{tabular}{|c|c|c|c|c|c|c|c|c|c|c|c|c|}
\hline \\
\hline Code & $\mathbf{N}^{\circ}$ & $\underset{\left(\mathrm{km}^{2}\right)}{\mathrm{S}}$ & $\begin{array}{l}\overline{V C n}_{d=1} \\
\text { observé } \\
\left(\mathrm{m}^{3} / \mathrm{s}\right)\end{array}$ & Code & $\mathbf{N}^{\circ}$ & $\underset{\left(\mathrm{km}^{2}\right)}{\mathrm{S}}$ & $\begin{array}{c}\overline{v c n}_{d=1}[1] \\
\text { observé } \\
\left(\mathrm{m}^{3} / \mathrm{s}\right)\end{array}$ & $\begin{array}{c}\overline{v c n}_{d=1}[2] \\
\text { régression }(12) \\
\left(\mathrm{m}^{3} / \mathrm{s}\right)\end{array}$ & $\begin{array}{c}\overline{v c n}_{d=1}[3] \\
\text { jaugeages } \\
\text { épisodiques } \\
\left(\mathrm{m}^{3} / \mathrm{s}\right) \\
\end{array}$ & K & $\begin{array}{c}\text { Écart relatif } \\
([1]-[2]) /[1] \\
(\%)\end{array}$ & $\begin{array}{c}\text { Écart relatif } \\
([1]-[3]) /[1] \\
(\%)\end{array}$ \\
\hline A511061* & 10 & 1985 & 6,06 & A543101 & 14 & 963 & 1,11 & 1,40 & 1,04 & 0,171 & -26 & 6 \\
\hline A600101* & 17 & 66,5 & 0,428 & A605102 & 18 & 371 & 1,59 & 1,31 & 1,52 & 3,55 & 18 & 4 \\
\hline$A 712201^{*}$ & 29 & 226 & 0,084 & A782101 & 34 & 928 & 1,03 & 0,919 & 0,772 & 9,19 & 11 & 25 \\
\hline
\end{tabular}

Sous-bassin étudié Sous-bassin étudié
(jeu de validation) 


\subsection{Lien entre loi régionale des modules (3) et loi régionale des étiages (11)}

Nous observons (figure 8) une convergence et quasi-superposition des deux lois régionales pour les quantiles secs $\left(U_{F}<0,693\right)$. Cette observation est intéressante puisqu'elle implique pour les quantiles secs un choix indifférencié de la loi régionale, à la norme près $\overline{q a}_{\text {site cible }}$ pour les modules et $\overline{v C n}_{d=1 \text {, site cible }}$ pour les étiages.

II semble donc exister une certaine dépendance entre les modules annuels et les étiages pour les années moyennes à sèches. Si cette dépendance est tant soit peu effective, notre démarche simplifiée de la méthode des jaugeages épisodiques devrait être vérifiée pour estimer le module médian $\overline{q a}$. Autrement dit, nous reconduisons les tableaux 7 et 8 pour les modules annuels (tableaux 9 et 10). Pour un premier commentaire des résultats de ces tableaux nous reprendrons les cinq points retenus pour les étiages qui toutes choses considérées sont plus mitigés pour les modules. II est vrai que l'imprécision liée à (5) est en général plus faible que celle relative à (12).

Néanmoins, le coefficient $k$ de tendance établi pour les étiages permet une estimation cohérente du module médian $\overline{q a}$, par comparaison à l'observé et à (5). Ce résultat intéressant laisse supposer un lien entre régimes des modules annuels et d'étiage des sous-bassins de la Moselle française pour les années moyennes à sèches.

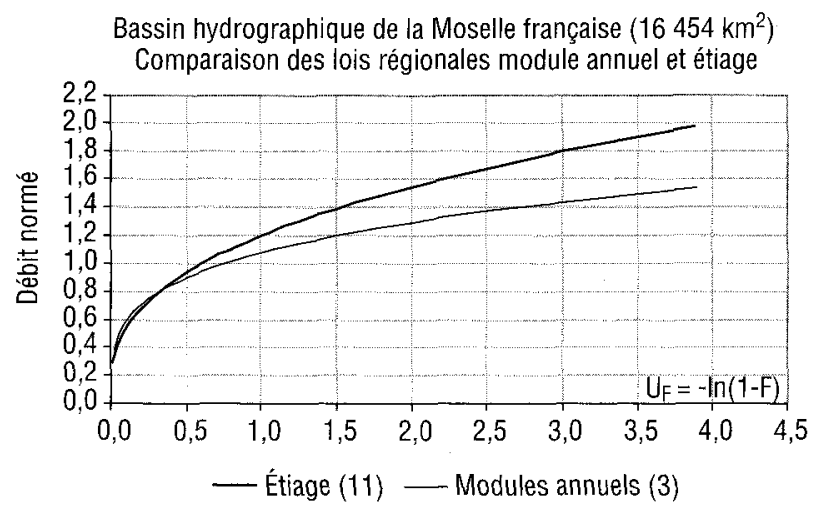

Figure 8 Comparaison des modèles régionaux.

Comparison of regional models. 
Tableau 9 Estimation du descripteur de régime $\overline{q a}$ selon (5) ou selon l'approche jaugeages épisodiques.

A415201* : sous-bassin de référence de proximité.

A614201 : peu (pas) d'incidence des étiages influencés sur le module.

Table 9 Estimation of regime descriptor $\overline{q a}$ according to (5) or to the episodic gauging approach.

A415201*: proximity of the reference sub-basin.

A614201: a small influence on the low-flow without significant effect on the annual mean discharge.

\begin{tabular}{|c|c|c|c|c|c|c|c|c|c|c|c|c|}
\hline \multicolumn{4}{|c|}{$\begin{array}{l}\text { Sous-bassin de référence } \\
\text { (jeu de calage) }\end{array}$} & \multicolumn{9}{|c|}{$\begin{array}{l}\text { Sous-bassin étudié } \\
\text { (jeu de validation) }\end{array}$} \\
\hline Code & $\mathbf{N}^{\circ}$ & $\underset{\left(\mathbf{k m}^{2}\right)}{\mathrm{S}}$ & $\begin{array}{c}\overline{q a} \\
\text { observé } \\
\left(\mathrm{m}^{3} / \mathrm{s}\right)\end{array}$ & Code & $\mathbf{N}^{\circ}$ & $\underset{\left(\mathrm{km}^{2}\right)}{\mathrm{S}}$ & $\begin{array}{c}\overline{q a}[1] \\
\text { observé } \\
\left(\mathrm{m}^{3} / \mathrm{s}\right)\end{array}$ & $\begin{array}{c}\overline{g a}[2] \\
\text { estimé } \\
\text { par régression (5) } \\
\left(\mathrm{m}^{3} / \mathrm{s}\right) \\
\end{array}$ & $\begin{array}{c}\overline{q a}[3] \\
\text { estimé } \\
\text { par jaugeages } \\
\left(\mathrm{m}^{3} / \mathrm{s}\right) \\
\end{array}$ & K & $\begin{array}{c}\text { Écart relatif } \\
([1]-[2]) /[1] \\
(\%)\end{array}$ & $\begin{array}{c}\text { Écart relatif } \\
([1]-[3]) /[1] \\
(\%)\end{array}$ \\
\hline A415201* & 2 & 186 & 8.57 & A405062 & 1 & 153 & 6.99 & 5.59 & 6.00 & 0.70 & 20 & 14 \\
\hline A436202* & 7 & 357 & 10,2 & A425064 & 5 & 1222 & 39,1 & 35,2 & 39,4 & 3,86 & 10 & 0 \\
\hline A444201 & 8 & 145 & 1,92 & A524201 & 11 & 116 & 1,55 & 1,58 & 1,58 & 0,82 & 2 & 2 \\
\hline A444201 & 8 & 145 & 1,92 & A525101 & 12 & 399 & 4,75 & 5,24 & 5,91 & 3,08 & -10 & -24 \\
\hline A444201 & 8 & 145 & 1,92 & A530301 & 13 & 64 & 0,740 & 0,830 & 0,924 & 0,48 & -12 & -24 \\
\hline A614201 & 19 & 152 & 3,59 & A605102 & 18 & 371 & 8,21 & 10,7 & 7,14 & 1,99 & -30 & 13 \\
\hline A656111 & 23 & 567 & 6,64 & A543101 & 14 & 963 & 10,8 & 11,8 & 7,90 & 1,19 & -9 & 27 \\
\hline A656111* & 23 & 567 & 6,64 & A673122 & 24 & 499 & 5,92 & 6,86 & 8,50 & 1,28 & -16 & -44 \\
\hline A735201* & 31 & 366 & 3,49 & A721301 & 30 & 38,4 & 0,373 & 0,470 & 0,265 & 0,08 & -26 & 29 \\
\hline A764201 & 33 & 144 & 1,32 & A782101 & 34 & 928 & 7,61 & 7,64 & 5,72 & 4,33 & 0 & 25 \\
\hline A788101 & 36 & 1273 & 9,65 & A975201 & 57 & 495 & 3,64 & 4,50 & 3,95 & 0,41 & -24 & -9 \\
\hline A885301 & 45 & 47 & 0,425 & A994201 & 59 & 1148 & 10,9 & 10,6 & 9,99 & 23,5 & 3 & 8 \\
\hline
\end{tabular}


Tableau 10 Estimation du descripteur de régime $\overline{q a}$ selon (5) ou selon l'approche jaugeages épisodiques.

A511061* : sous-bassin de référence de proximité.

A600101 : peu (pas) d'incidence des étiages influencés sur le module.

Table 10 Estimation of regime descriptor qa according to (5) or to the episodic gauging approach.

$A 511061^{*}$ : proximity of the reference sub-basin.

A600101: a small influence on the low-flow without significant effect on the annual mean discharge.

\begin{tabular}{|c|c|c|c|c|c|c|c|c|c|c|c|c|}
\hline \multicolumn{4}{|c|}{$\begin{array}{c}\text { Sous-bassin de référence } \\
\text { (jeu de validation complémentaire) }\end{array}$} & \multicolumn{9}{|c|}{$\begin{array}{l}\text { Sous-bassin étudié } \\
\text { (jeu de validation) }\end{array}$} \\
\hline Code & $\mathbf{N}^{\circ}$ & $\underset{\left(\mathrm{km}^{2}\right)}{\mathrm{S}}$ & $\begin{array}{c}\overline{q a} \\
\text { observé } \\
\left(\mathrm{m}^{3} / \mathrm{s}\right)\end{array}$ & Code & $\mathbf{N}^{\circ}$ & $\underset{\left(\mathrm{km}^{2}\right)}{\mathrm{S}}$ & $\begin{array}{c}\overline{q a}[1] \\
\text { observé } \\
\left(\mathrm{m}^{3} / \mathrm{s}\right)\end{array}$ & $\begin{array}{l}\overline{q a}[2] \\
\text { régression }(5) \\
\left(\mathrm{m}^{3} / \mathrm{s}\right)\end{array}$ & $\begin{array}{c}\overline{q a}[3] \\
\text { jaugeages } \\
\text { épisodiques } \\
\left(\mathrm{m}^{3} / \mathrm{s}\right) \\
\end{array}$ & K & $\begin{array}{c}\text { Écart relatif } \\
([1]-[2]) /[1] \\
(\%)\end{array}$ & $\begin{array}{c}\text { Écart relatif } \\
([1]-[3]) /[1] \\
(\%)\end{array}$ \\
\hline AA511061* & 10 & 1985 & 47,9 & A543101 & 14 & 963 & 10,8 & 11,8 & 8,19 & 0,171 & -9 & 24 \\
\hline A600101* & 17 & 66,5 & 2,15 & A605102 & 18 & 371 & 8,21 & 10,7 & 7,63 & 3,55 & -30 & 7 \\
\hline A712201* & 29 & 226 & 1,35 & A782101 & 34 & 928 & 7,61 & 7,64 & 12,4 & 9,19 & 0 & -63 \\
\hline
\end{tabular}




\section{6 - CONCLUSION}

Les modélisations régionales proposées sur les modules annuels et les étiages des sous-bassins de la Moselle française apportent une connaissance hydrologique nécessaire à la gestion intégrée des ressources en eau. Elles s'inscrivent dans le contexte des directives de la loi Pêche et de la loi sur l'Eau et plus récemment de la Directive Cadre Européenne. Le concept débit-duréefréquence $(Q d F)$ appliqué ces dernières années aux étiages permet de rendre compte du régime hydrologique d'étiage. II est fort vraisemblable, dans un avenir proche, que la référence QMNA5 ${ }^{1}$ ne suffira plus pour satisfaire à une gestion intégrée de la ressource en eau des bassins versants. Une connaissance multi-fréquences et multi-durées des étiages sera sans doute nécessaire pour satisfaire à la fois la complexité des usages de l'eau et la préservation des hydrosystèmes.

Le caractère opérationnel de ces modélisations régionales dépend essentiellement de la précision d'estimation des descripteurs de régime du site cible, $\overline{q a}$ pour les modules annuels et $\overline{v c n}_{d=1}$ ainsi que $\Delta \mathrm{e}$ dans une moindre mesure pour les étiages. Pour $\overline{q a}$ le modèle d'estimation (5) explique $98 \%$ de variance et son incertitude est de l'ordre de $19 \%$ pour un intervalle de confiance à $70 \%$. Pour $\overline{v C n}_{d=1}$ l'estimation donnée par (12) est moins précise, d'environ $35 \%$ (intervalle à $70 \%$ ) pour une variance expliquée de $93 \%$. II faut cependant relativiser cette imprécision dans la mesure où ce descripteur est un débit d'étiage généralement faible. C'est plutôt une cohérence de l'ordre de grandeur d'estimation $\overline{v c n}_{d=1}$ qui est à rechercher en associant en particulier le modèle d'estimation (12) et la méthode simplifiée des jaugeages épisodiques. Cette dernière donne des estimations réalistes et à défaut pour l'instant de résultats géostatistiques probants (BLUM, 2003) elle peut représenter une alternative (ou complémentarité) intéressante aux méthodes de régionalisation à bases géostatistiques. Nécessitant un faible investissement en mesures de débit des sous-bassins non observés, par rapport au réseau national de suivi hydrométrique, elle peut être facilement mise en œuvre par exemple au niveau des Syndicats de Bassin. La précision obtenue pour $\Delta e$ (13) reste suffisante malgré son incertitude d'environ $45 \%$ (intervalle à $70 \%$ ). En effet, l'objectivité des quantiles de différentes durées modélisés est conservée (GALÉA et al., 1999 ; CANALI, 2002) du fait du caractère linéaire de la fonction $z(d)$ associée à $\Delta e(11)$. Une pseudo dépendance est observée entre modules annuels et débits d'étiage pour les années moyennes à sèches. Cette pseudo-dépendance reste à confirmer pour d'autres bassins hydrographiques. Elle permet une démarche simple commune à partir de quelques jaugeages épisodiques pour conforter (ou corriger) l'ordre de grandeur d'estimation des descripteurs de débit $\overline{q a}$ (5) ou $\overline{v C n}_{d=1}$ (12). Cela est d'autant plus vrai que l'écoulement est peu ou prou influencé, dans ce cas en effet les modèles d'estimation (5) et (12) peuvent se révéler peu performants.

L'ensemble des connaissances relatives à cette recherche est repris dans un Système d'Information Géographique pour répondre éventuellement à la demande (VANDERBECQ, 2003).

1. QMNA5 : débit mensuel minimum annuel quinquennal « $\sec »$. 


\section{RÉFÉRENCES BIBLIOGRAPHIQUES}

BLUM M., 2003. La détermination de régions hydrologiques homogènes par l'analyse de corrélation canonique. DESS SITN, soutenu le 17 septembre 2003 à l'Université Claude Bernard, Lyon 1. UR hydrologie-Hydraulique, Cemagref Groupement de Lyon.

CANALI S., 2002. Régionalisation des régimes d'étiage et des modules annuels des sous-bassins de la Moselle française. DESS, Université de Tours (octobre 2002).

Developments in water science 48, 2003. Hydrological Drought, processes and estimation methods for streamflow and groundwater. Editors : Lena M. TALIAKSEN, Henny A.J. VAN LANEN - 24 February 2003.

Directive 2000/60/CE du Parlement Européen et du Conseil du 23 octobre 2000 établissant un cadre pour une politique communautaire dans le domaine de l'eau, Journal officiel des Communautés européennes, 22/12/2000, L 327, pp. 1-72.

Directive 2000/60/CE du Parlement Européen et du Conseil du 23 octobre 2000 établissant un cadre pour une politique communautaire dans le domaine de l'eau, Procédure d'élaboration de l'état des lieux : caractérisation du district hydrographique et registre des zones protégées, Organisation des travaux 2002-2004, ministère de l'Aménagement du Territoire et de l'Environnement, Direction de l'eau, document du 06/02/2002, $48 \mathrm{p}$.

DUSSAUX V., 2000. Modèles débit-duréefréquence des régimes d'étiage, modèles de conceptualisation adaptée aux distributions fréquentielles les plus utilisées. Mémoire de fin d'études, ENTPE Lyon, $48 \mathrm{p}$.

GALÉA G., MERCIER G.X. ET ADLER M.J*, 1999. Modèles débit-durée-fréquence d'étiage, concept et usage pour une approche régionale des régimes de basses eaux des bassins hydrographiques de la Loire (France) et du CrisuAlb (Roumanie). Cemagref-Lyon, Division Hydrologie Hydraulique - (*) I.N.M.H. Bucarest, Roumanie/Revue des Sciences de l'Eau, 12/1 (1999) 93-122.

GALÉA G., JAVELLE P., CHAPUT N., 2000. Un modèle débit-durée-fréquence pour caractériser le régime d'étiage d'un bassin versant. Revue des sciences de l'eau, 13(4), pp. 421-440.

GALÉA G., GENDREAU N., JAVELLE P., POINARD D., PUECH C., SCHNEIDER M., 2001 : Régimes des crues des sousbassins de la Moselle française / modèles régionaux et SIG associé. Projet PACTES, UR Hydrologie-Hydraulique, Cemagref-Lyon, ocobre 2001.

JAVELLE P., 2001. Caractérisation du régime des crues. Le modèle débit-durée-fréquence convergent - Approche locale et régionale. UR Hydrologie-Hydraulique, Cemagref, groupement de Lyon, thèse soutenue le 01.03.2001 à l'INPG (MMGE).

JAVELLE P., OUARDA T.B.M.J., LANG M., BOBÉE B. GALÉA G., GRÉSILLON J-M., 2002. Development of regional flowduration-frequency curves based on the index-flood method, Journal of Hydrology 258 (2002) 249-259.

NOAH, 2000. New Opportunities for Altimetry in Hydrology - Final Report, European Commission DG XII, Environment and Climat, Theme 3, Space and Techniques, CEO, ref. NOAH ENV4-CT96-0371, $318 p$.

OBERLIN G, GALEA G., TONI J., 1973. Intérêt des jaugeages épisodiques pour l'estimation des étiages des petits bassins non équipés, Cahier ORSTOM série hydrologie, 10 (4), pp. 349-368.

PACTES, 2001. Projet PACTES/Prévention, dossier de travail. UR HydrologieHydraulique, Cemagref, groupement de Lyon, 2 mars 2001.

PACTES, 2001. PACTES Module PREVENTION, Séminaire prévention Montpellier, 5-6 juin 2001. UR Hydrologie-Hydraulique, Cemagref, groupement de Lyon.

SAUQUET E., LEBLOIS E. ET GALÉA G., 2003. Mapping flood regime descriptors for flow-duration-frequency characterisation at ungauged location. EGS-2003 (Nice)/poster.

VANDERBECQ A., 2003. Réalisation d'une application métier S.I.G. Intégration du modèle QdF sous Arc View 3.2. DESS : Application de l'informatique aux Sciences de la Terre, soutenu le 23.10.2003 à l'ENSG, Marne la Vallée. 